\title{
EL MODELO DE EVALUACIÓN PARTICIPATIVO CONTEXTUALIZADO (MEPAC) APLICADO AL PROYECTO “GRANADA-EMPLEO”
}

\author{
THE CONTEXTUALIZED PARTICIPATIVE MODE OF EVALUATION (CPME) \\ APPLIED TO “GRANADA-EMPLOYMENT” PROJECT
}

\author{
Margarita Pérez Sánchez \\ Antonio Trinidad Requena \\ Nieves Ortega Pérez \\ Universidad de Granada. España/Spain \\ mperezs@ugr.es \\ atrinida@ugr.es \\ ortega@ugr.es
}

Recibido/Received: 18/01/2013

Modificado/Modified: 19/02/2013

Aceptado/Accepted: 20/06/2013

\section{RESUMEN}

Este artículo muestra la evaluación del Proyecto "Granada-Empleo", subvencionado por el Fondo Social Europeo y coordinado por la Diputación de Granada de 2009-2010.

La evaluación que aquí se expone -realizada de septiembre de 2010 a julio de 2011 - ha sido diseñada por un equipo de investigadores del Departamento de Ciencia Política y de la Administración y de Sociología de la Universidad de Granada. Este trabajo aporta un ejemplo aplicado del "Modelo de Evaluación Participativo Contextualizado" (MEPAC), diseñado ad hoc por el equipo externo.

La evaluación sumativa del proyecto se expone siguiendo los objetivos principales que persigue y las medidas que lo desarrolla, revelando claves en el desarrollo futuro de pactos territoriales por el empleo y actividades dirigidas a la mejora de empleo de los colectivos demandantes de trabajo.

\section{PALABRAS CLAVE}

Evaluación, desempleo, formación ocupacional, inserción laboral, pacto territorial.

\section{SUMARIO}

1. El Proyecto "Granada-Empleo" como política activa de empleo. 2. La evaluación externa de resultados del Proyecto "Granada-Empleo". 2.1. El "Modelo de Evaluación Participativo Contextualizado" (MEPAC). 2.2. El proceso y técnica de evaluación del Proyecto "Granada-Empleo". 3. Evaluación de resultados del Programa "Granada-Empleo". 3.1. La evaluación del impulso y consolidación de los pactos territoriales de empleo. 3.2. La implementación de itinerarios integrados de inserción sociolaboral. 4. Conclusiones. Bibliografía.

\section{ABSTRACT}

This article presents the evaluation of 2009-2010 "Granada-Employment" Project financed by the European Social Fund and coordinated by the Granada County Council. The evaluation -developed between September of 2010 and July of 2011- was designed by the Departments of Political Science and Public Administration, and Sociology from the University of Granada. This research offers a 
Contextualized Participative Mode of Evaluation (CPME) case study, which is ad hoc designed by the external evaluation team.

The summative evaluation of the Project undergoes the main objectives pursed and the measures developed by the programme, and it reveals key points for the territorial pacts future development for employment and actions addressed to improve the job seekers' labour integration.

\section{KEYWORDS}

Evaluation, unemployment, vocational training, labour integration, territorial pact.

\section{CONTENTS}

1. The "Granada-Employment" Project as active employment policy. 2. The external result evaluation of the "Granada-Employment" Project. 2.1. The Contextualized Participative Mode of Evaluation (CPME). 2.2. The evaluation process and technique of the "Granada-Employment" Project. 3. "Granada-Employment" programme result evaluation. 3.1. The evaluation of the promotion and consolidation of the territorial pacts for employment. 3.2. The implementation of the social and labour integration tracks. 4. Conclusions. References.

\section{EL PROYECTO "GRANADA-EMPLEO" COMO POLÍTICA ACTIVA DE EMPLEO}

Este trabajo expone el seguimiento y la evaluación del Proyecto "Granada-Empleo", subvencionado por el Fondo Social Europeo y coordinado por el Área de Cultura, Juventud y Desarrollo Local de la Diputación de Granada 2009-2010.

La evaluación que aquí se presenta -realizada de septiembre de 2010 a julio de 2011 y financiada por la Delegación de Promoción Económica y Empleo de la Diputación de Granada- es entendida como una oportunidad de análisis y mejora que se realiza desde la óptica de la retroalimentación con la transversalidad de género, y con una metodología participativa y una pluralidad de técnicas y herramientas.

Este trabajo ha sido realizado por un equipo de investigadores del Departamento de Ciencia Política y de la Administración y de Sociología de la Universidad de Granada, y aporta un ejemplo aplicado de modelo de evaluación diseñado ad hoc por el equipo externo.

El "Modelo de Evaluación Participativo Contextualizado" (MEPAC) es un modelo que permite la participación activa de los actores implicados en un primer momento al determinar qué evaluar, y su implicación en el juicio de valor final de los resultados en un tercer nivel. Así, se busca que los valores e intereses de los implicados se recojan en el modelo evaluativo, y asegurar la pluralidad de perspectivas de los actores, sus objetivos y expectativas con respecto al Programa.

El Proyecto "Granada-Empleo" es diseñado por la Diputación Provincial de Granada como una estrategia de intervención en materia sociolaboral, consensuado y coordinado entre las distintas entidades, dirigida a la mejora del funcionamiento del mercado de trabajo y de las posibilidades de empleo de la población en desventaja de la provincia. El programa se orienta hacia actividades de inserción laboral, como los pactos territoriales por el empleo, y políticas de formación; este abarca 166 municipios de la provincia de Granada, que concentran el $65,6 \%$ de la población total de la provincia y representan el $97,5 \%$ de la superficie provincial. 
Esta área de intervención del Programa presenta elevadas tasas de inactividad, y desempleo de larga y muy larga duración. Los grupo-objetivo del Programa "GranadaEmpleo" son mujeres desempleadas, jóvenes, mayores de 45 años, y personas desempleadas de larga duración. También se consideran entre los colectivos prioritarios, aquellos en riesgo de exclusión social, como son los cuidadores/as de personas dependientes, inmigrantes y minorías étnicas, personas con discapacidad, jóvenes que no han superado la enseñanza obligatoria, víctimas de violencia de género, ex toxicómanos/as y ex reclusos/as.

El objetivo general del Programa "Granada-Empleo" es: "diseñar, implantar y ejecutar una estrategia de intervención en el marco de planificaciones integrales dirigidas a mejorar la situación sociolaboral de las personas en situación o riesgo de exclusión, en materia sociolaboral, de forma coordinada con el resto de entidades y agentes socioeconómicos que intervienen en el territorio".

El Programa aborda siete áreas de intervención -desarrolladas a partir de veintidós medidas- que son objeto del estudio de la evaluación externa que aquí se presenta. La primera es la destinada a impulsar y consolidar los pactos territoriales para el empleo. La segunda es la puesta en marcha y gestión del laboratorio de empleo a través del Observatorio Provincial de Empleo. Además, busca implementar, en tercer lugar, itinerarios integrados de inserción sociolaboral, y también persigue el fomento del uso de las nuevas tecnologías. La quinta área de actuación es el seguimiento y evaluación del propio Programa; la sexta son acciones de sensibilización, información y difusión de la metodología, los resultados y las buenas prácticas; y por último, tiene como ámbito de actuación las redes de intercambio de conocimientos y buenas prácticas.

\section{LA EVALUACIÓN EXTERNA DE RESULTADOS DEL PROYECTO "GRANADA-EMPLEO"}

Este trabajo se centra en la evaluación de los resultados. Esta se relaciona con los conceptos de eficacia y efectividad del Programa. Analiza el cumplimiento de los objetivos en sus dimensiones de cantidad, calidad y tiempo, los efectos del proyecto no programados -positivos o negativos- sobre la población beneficiaria y objeto del mismo, y la opinión y grado de satisfacción de los beneficiarios del programa. Con el fin de comprobar la influencia de los factores externos sobre los resultados, se lleva a cabo un análisis comparativo entre el grupo beneficiario de programa y un "grupo de comparación", compuesto por aquellos que se presentaron como potenciales participantes y no fueron seleccionados. Tanto en la evaluación de la implementación como en la de resultados, se han tenido en cuenta la perspectiva de género y las actuaciones en materia de igualdad.

Uno de los elementos clave de este trabajo es el diseño de un modelo ad hoc, denominado "Modelo de Evaluación Participativo Contextualizado" (MEPAC) para la realización de la evaluación.

\subsection{El "Modelo de Evaluación Participativo Contextualizado" (MEPAC)}

El tipo de evaluación aplicado al Proyecto "Granada-Empleo" fue una evaluación formativa y sumativa en cuanto que se evaluó durante el transcurso del Programa y al final del mismo; una evaluación externa, en cuanto a la procedencia del grupo evaluador, en este caso de la Universidad de Granada; y por último, y en cuanto a la naturaleza de lo 
evaluado, fue una evaluación de implementación y de resultados, ya que analizó tanto el comportamiento y los procesos como el resultado final del proyecto.

La evaluación constó de cinco sucesivos cortes evaluativos. El primero duró tres meses, y se negoció y consensuó, con las entidades presentes en el territorio que se han sumado a este proyecto (30 en total: Mancomunidades, Consorcios, Agentes socioeconómicos, etc.), tanto las dimensiones evaluativas, el sistema de indicadores, como la cuantificación de los resultados previstos. El segundo, tercero y cuarto corte se dedicaron a la recogida de datos y redacción de consejos operativos de retroalimentación en la fase de implementación del proyecto. En el quinto y último corte evaluativo se efectuó el trabajo de campo y la recogida de datos sobre los resultados finales y la redacción del informe final de evaluación.

Los principios que subyacen en este novedoso modelo (desarrollado por el equipo de investigación de la Universidad de Granada que presenta este proyecto) se basan en la participación activa de los actores implicados en el Programa "Granada-Empleo" en varios de los niveles del modelo. Esta participación implica la negociación y consenso en el primer nivel del modelo, donde se determina el qué evaluar, y su implicación en el juicio de valor final de los resultados en el tercer nivel. Con ello se consigue que los valores e intereses de los implicados se recojan en el modelo evaluativo, así como asegurar la pluralidad de perspectivas de los actores, sus objetivos y expectativas con respecto al Programa. Este modelo se construye desde el enfoque constructivista, participativo y plural, donde el dialogo con los actores claves es el punto de partida para el diseño posterior (niveles).

El modelo se desarrolla en tres niveles: negociación, descripción y valoración o juicio de valor. El nivel 1 denominado "NEGOCIACIÓN" implica la participación de los responsables en sesiones de trabajo conjunto de la ejecución, el equipo evaluador y del personal técnico en la negociación de las dimensiones evaluativas, el sistema de indicadores, y la cuantificación de resultados previstos que se va a utilizar en la evaluación del proyecto, en definitiva el qué evaluar. Los indicadores negociados abordan los aspectos básicos del programa: indicadores de realización (para medir el avance de la ejecución), de resultados (que miden los efectos directos o inmediatos, la calidad o el rendimiento que la realización de la acción consigue), y de impactos (de las acciones realizadas y los resultados obtenidos). También en este punto del proceso se pueden pactar indicadores complementarios -a partir de los anteriores- y de eficacia (para medir lo que se ha conseguido con relación a lo que se pretendía).

El segundo nivel denominado "DESCRIPCIÓN" busca conocer los procesos de implementación -servicios concretos, cobertura y dinámica interna- y resultados del Programa. Para ello utilizamos la técnica de la triangulación tanto de métodos como de sujetos, con la intención de aumentar la fiabilidad de los resultados. En el caso de la triangulación de métodos utilizamos para recoger la misma información las memorias de actividades del Programa, las entrevistas personales al personal técnico del Programa y encuestas al personal usuario del mismo. En relación a la triangulación de sujetos tenemos en cuenta la posición del personal técnico, del personal usuario y las entidades y organizaciones participantes y ejecutoras del proyecto. En este estadio se miden las dimensiones e indicadores establecidos en el primer nivel. En este nivel intermedio revierte gran importancia el contexto en el que se aplica el Programa, este es las entidades territoriales como variable que puede incidir en la implementación y resultados del Programa. 


\section{FIGURA 1. MODELO DB EVALUACIÓN PARTICIPATIVO CONTEXTUALIZADO 1 NIVEL: NEGOCIACIÓN}

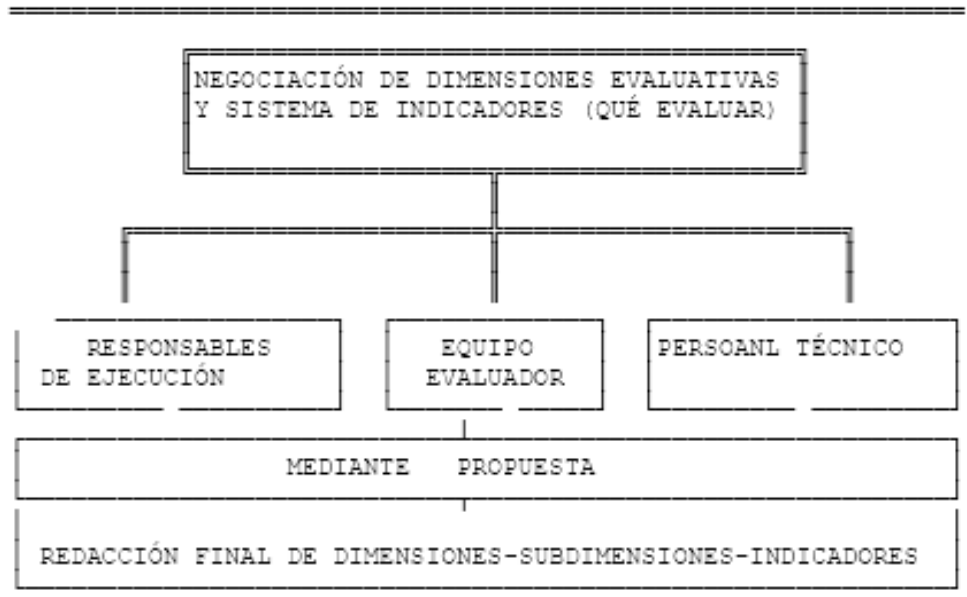

2 NIVEL: DESCRIPCIÓN

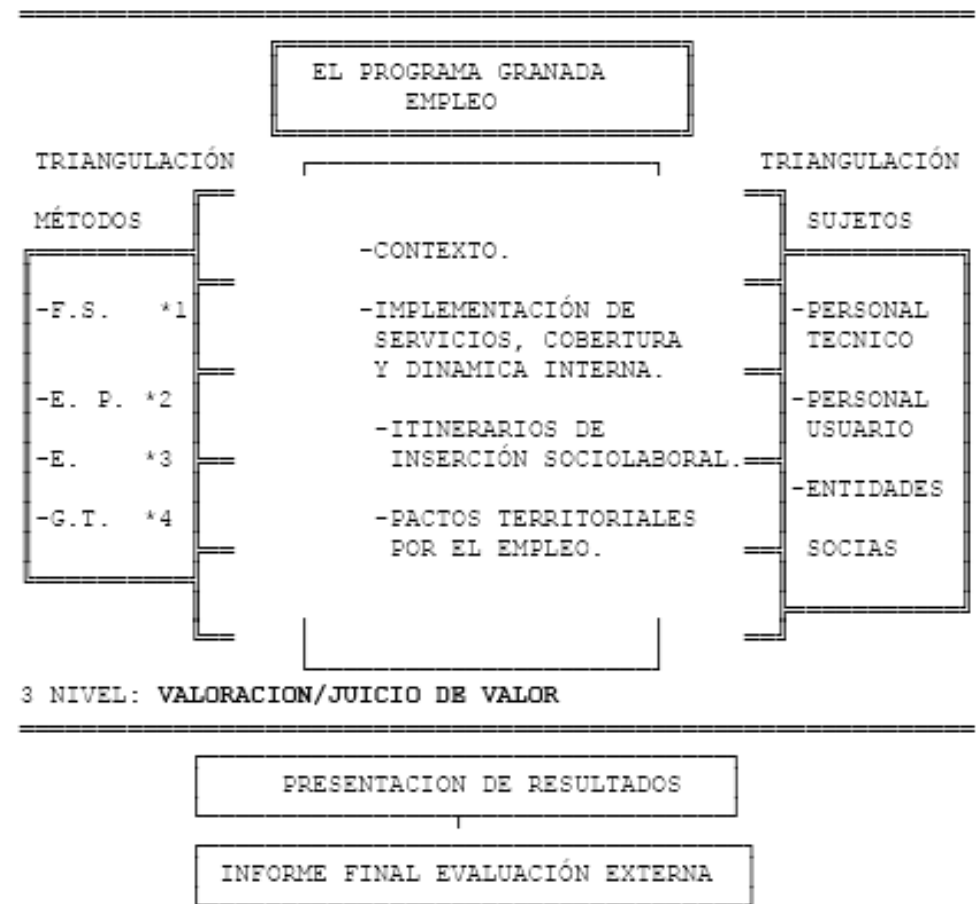

*1.Ficha de seguimiento. *2. Entrevistas personales. *3. Encuestas. *4.Grupo de trabajo con equipos técnicos.

Fuente: Elaboración propia a partir de Trinidad y Pérez (2010:188)

En el tercer y último nivel, denominado "VALORACION/JUICIO DE VALOR", los agentes implicados en el Programa toman protagonismo y participan en el juicio de valor 
que implica toda evaluación. En este sentido presentamos un avance de resultados en sesiones de trabajo, donde se contextualizan y ponderan los resultados en función de las valoraciones de los actores interesados y la cuantificación de los resultados previstos y negociados en el nivel 1 del modelo.

\subsection{El proceso y técnica de evaluación del Proyecto "Granada-Empleo"}

El proceso de evaluación ha incluido un amplio trabajo técnico que incluye entrevistas semiestructuradas, encuestas, revisión documental y la creación y aplicación de los indicadores de implementación y resultados creados para esta evaluación.

Se han realizado un total de 37 entrevistas individuales semiestructuradas durante dos meses de aplicación (abril y mayo de 2011) a actores políticos y técnicos participantes en el programa. Las entrevistas fueron seleccionadas atendiendo a la tipología de comarca confeccionada por el equipo de evaluación externa. Esta se ha hecho teniendo en cuenta el tamaño del municipio y su distribución (dispersión y posición socioeconómica en el territorio) geográfica en la comarca.

Con el objetivo de conocer las opiniones de las personas beneficiarias -terminaron el curso de formación-, los que abandonaron el curso de formación (después de al menos un mes), y los no beneficiarios -no admitidos en el curso de formación- se encargó al Centro de Análisis y Documentación Política y Electoral de Andalucía (CADPEA) la aplicación de una encuesta telefónica. El trabajo de campo fue realizado entre el 5 y el 7 de abril de 2011 por el laboratorio de encuestas telefónicas asistidas por ordenador (sistema CATI) de la Universidad de Granada.

En cuanto a la revisión documental, como materiales producidos dentro del Programa, se ha trabajado con la evaluación de las oficinas territoriales, el informe actualizado sobre los pactos, el documento con las principales tareas y funciones realizadas por el equipo de agentes dinamizadores/as territoriales, los informes de ejecución y de publicidad trimestrales 2008-2010, el Plan inicial de información y publicidad, el proyecto "Granada-Empleo", mapeos relacionales de los doce pactos territoriales, planes operativos anuales (POAs) de los mismos, y el programa operativo "Fondo Social Europeo. 2007-2013 - Andalucía" y sus instrucciones de aplicación y desarrollo.

Finalmente, la relación de indicadores creados conforma un total de 172 de implementación y 90 de resultados. Estos fueron consensuados en siete reuniones grupales con el equipo técnico y agentes dinamizadores de "Granada-Empleo", llevadas a cabo en cada territorio.

\section{EVALUACIÓN DE RESULTADOS DEL PROGRAMA “GRANADA-EMPLEO"}

De los objetivos del programa mencionados anteriormente, en este estudio nos centraremos en dos de ellos, que corresponden a los instrumentos tradicionales de las políticas activas de empleo; estos son la consolidación de los pactos territoriales para el empleo y la implementación de itinerarios integrados de inserción laboral. A continuación, se presentan los resultados de la evaluación.

\subsection{La evaluación del impulso y consolidación de los pactos territoriales de empleo}

Este objetivo se ve desarrollado a partir de siete medidas distintas. La primera de las medidas concretada en la elaboración de una metodología de trabajo específica detecta el 
asentamiento de metodologías y formas de trabajo ya asentadas de los actores locales a partir de la diagnosis de las necesidades. Esta estrategia genera confianza en la planificación y mejora el conocimiento del terreno.

En cuanto a la estrategia de intervención se han dejado mayoritariamente de utilizar herramientas colectivas de trabajo (reuniones grupales con las organizaciones involucradas) y se tiende a la aplicación de herramientas individuales, lo que responde mejor a la naturaleza de los trabajos, las distancias geográficas internas de algunos territorios y las agendas de trabajo de técnicos y políticos.

Una de las barreras que tuvieron que superar en la primera etapa los dinamizadores en los territorios, fue la de desligar su figura a un partido político específico, lo que una vez conseguido consolidaba el carácter independiente del programa.

Las líneas estratégicas más repetidas en los distintos planes se refieren a turismo, actuaciones administrativas, actividades económicas, formación e integración comarcal y colaboración. En contrapartida, algunas de las líneas que menos están presentes son las relacionadas con el comercio, la dinamización empresarial y juventud.

Desde la perspectiva del cumplimiento de las necesidades reales del territorio, se detecta en los discursos de los actores locales una falta de concreción en las acciones emprendidas directamente relacionada con la falta de presupuesto y recursos propios del Programa. Se valora el esfuerzo teórico por conocer el territorio, pero también se hace visible la necesidad de traducir todo esto a iniciativas concretas.

Se puede decir que el Programa "Granada-Empleo" se constituyó en un espacio de incipiente aprendizaje donde los actores políticos y técnicos tuvieron que ejercitar nuevas formas de planificación basadas en el consenso como técnica de llegar a acuerdos. Este programa coordina y dinamiza las iniciativas que ya existen en un territorio pero que son puestas en marcha de forma individual, y constituye equipos técnicos de trabajo que buscan articular y dinamizar los recursos que ya existen en un territorio. Este concepto de participación se traduce en la idea de trabajar de "abajo hacia arriba" pudiendo con ello rescatar una dinámica de participación que situase al actor político y técnico del territorio más cercano a los objetivos del Programa.

La segunda de las medidas que desarrolla este primer objetivo es la definición de una estructura organizativa. Esta se plantea en dos niveles interrelacionados: el primero es de gestión global o general, compuesto por un Consejo Directivo -encargado del impulso y seguimiento institucional del proyecto- y por el Equipo Técnico Central, que ejecuta la coordinación técnica de las acciones que se desarrollan en el marco del Programa. El segundo nivel es el territorial, que está ubicado en las distintas zonas de actuación, integrado por la "comisión institucional de zona" (CIZ), una "comisión técnica de zona" (CTZ), y los comités de ciudadanía.

En cuanto a la gestión a nivel global, los indicadores de implementación diseñados para esta medida señalan que se llevaron a cabo tres reuniones del Consejo Directivo en 2009, una presentación a nivel provincial del proyecto, y 20 reuniones de coordinación del Equipo Técnico. En cuanto al seguimiento, este se ha estructurado en torno a las reuniones de coordinación del Equipo Técnico, y de la coordinadora de equipos locales con las distintas oficinas territoriales, a través de cronogramas para la planificación de trabajo y calendarios para la entrega de tareas, y del uso de la página Web del Observatorio Territorial de Estudios y Análisis (OTEA) y correos electrónicos.

En relación a las capacidades de convocatoria del Programa, tanto los Agentes Territoriales como los Técnicos identifican como una ausencia sensible la de los Agentes 
Locales de Promoción y Empleo (en adelante ALPEs). Esta ausencia se ha subsanado por las relaciones informales que se fueron construyendo entre el equipo que está presente en los territorios y los ALPEs. No obstante, su ausencia, aunque vista como una carencia del Programa, no se ve como algo decisivo en el momento de llevar a cabo las actividades de "Granada-Empleo" en el territorio. La ausencia de esta relación formal se siente en el momento de establecer reciprocidad en los apoyos técnicos que hay sobre todo en los municipios más pequeños, donde los recursos humanos son más escasos.

En cuanto al nivel territorial, este se estructura como sigue: la comisión institucional compuesta por los ayuntamientos y mancomunidades y, en menor medida, por grupos de desarrollo rural, entidades privadas como empresas y cooperativas, sindicatos y asociacionesy las comisiones técnicas -compuestas por diferentes actores locales y territoriales en cada uno de los pactos-.

Las características del trabajo de los agentes territoriales muestra dos tendencias, por un lado, la importancia que adquiere el uso de las nuevas tecnologías en el trabajo de comunicación de los agentes con las entidades locales, como también el peso que adquiere el uso de las redes sociales en el trabajo local de los dinamizadores. Por otro lado, se detectó la constante labor de los agentes en el terreno local como complemento al uso de las nuevas formas de comunicación.

La comisión institucional se ha entendido por los agentes territoriales como una medida más del programa que reúne en una sola instancia a los representantes políticos de todo el abanico partidista, y es vista como una prestadora de servicios que no tiene paralelo en las entidades locales. Sin embargo, para los técnicos ajenos al programa, la figura de Diputación es considerada como lejana al territorio y a la implementación del Programa.

Por otro lado, la valoración que los diferentes agentes territoriales hacen sobre el equipo central y su trabajo de coordinación es mayoritariamente positiva.

La participación de las distintas realidades locales y la operatividad de los equipos en el terreno fueron los criterios en la conformación de las distintas comisiones técnicas. El alto nivel de participación en la configuración de las comisiones técnicas, aun cuando no siempre están formalizadas, fortalece el Programa y legitima sus estrategias de intervención y participación. La operatividad se traduce en que la comisión es un ente que permite la coordinación a nivel técnico con el fin de evitar redoblar esfuerzos. Aun entre los que consideran estas comisiones como una duplicidad de estructura creada en torno al "GranadaEmpleo", se entiende como un esfuerzo más genuino por tratar de llevar a la práctica desde la conformación de la propia estructura esa idea de trabajar "desde abajo hacia arriba", y valora el hecho de que se esté incidiendo en el propio territorio.

La elaboración del programa de promoción de concertación territorial es la tercera medida desarrollada de este primer objetivo. Esta medida perseguía incorporar al Programa a los líderes políticos, agentes socioeconómicos, el sector privado y al tejido asociativo presente en el territorio, a través de la figura de los Pactos y por medio de metodologías participativas. Por otra parte, también buscaba crear programas de participación ciudadana que incidan en la creación de una red de personas claves, la movilización del mayor número de asociaciones y colectivos presentes en el territorio, el diseño de procedimientos de participación dirigidos a los ciudadanos que no formen parte de dicho tejido, y mejorar la transparencia y eficacia de los canales de información que difunden los contenidos de carácter político.

En consonancia con lo que plantea el Programa, el discurso de los agentes territoriales destaca que el trabajo de la participación se ha hecho fundamentalmente a través del uso de las redes sociales, que optimizan al máximo los recursos económicos y de tiempo. 
En términos cualitativos la visión sobre los resultados de esta medida es dual. Por una parte, está presente el discurso que valora como significativo el uso de redes sociales como una herramienta legítima que permite la continuidad y permanencia a la participación. En cambio, para otros este tipo de iniciativas no logran el objetivo de la participación ciudadana. Ahora bien, si consideramos los cuatro aspectos donde el Programa pretendía incidir, el logro es más bien bajo. La red de personas claves no fue creada, no se visualizan acciones concretas que permitan afirmar que se ha producido una movilización del mayor número de asociaciones y colectivos de carácter ciudadano del territorio, y no hay referencias objetivas a procedimientos de participación dirigidos al ciudadano como individuo. El único aspecto donde quizá se logró incidir en alguna medida, a través del ya mencionado uso de las nuevas tecnologías, es el caso de mejorar la transparencia y eficacia de los canales de información. Por lo tanto, se advierte una descompensación entre lo que "Granada-Empleo" pretendía en materia de participación ciudadana y las herramientas, acciones y recursos que se articularon para conseguir dicho fin.

La medida cuarta persigue la constitución de doce comisiones técnicas de zona (CTZ) que tenían por finalidad la elaboración de estrategias de intervención en materia sociolaboral, de forma coordinada con el resto de las entidades que están presentes en el territorio. Sus áreas de trabajo se desarrollan tanto en el proceso de implementación de pactos como en los itinerarios integrados de inserción sociolaboral. Uno de los grandes logros del "GranadaEmpleo" fue aglutinar a la inmensa mayoría de municipios en la firma de pactos. Como se ha señalado anteriormente, participaron en el Proyecto 166 municipios y 11 entidades locales autónomas en la firma prevista de los doce pactos territoriales. En la iniciativa quedaron excluidos solo dos municipios de la provincia (Granada y Motril) que al ser entes de más de 50.000 habitantes tenían la posibilidad de acceder a otras ayudas.

En definitiva, el programa "Granada-Empleo" ha sido capaz de trabajar a un nivel intermedio, el comarcal, a partir del cual se puede visualizar la necesidad de tener un soporte institucional por arriba (provincial) y otro por abajo (municipal). Por otra parte, la escasez de tiempo y presupuesto es vista como una de las limitaciones relevantes a la hora de implementar el pacto tanto de actores políticos como de técnicos.

El programa de dinamizadores rurales o agentes territoriales es la quinta medida de este primer objetivo. El dinamizador es la figura técnica responsable de cada grupo territorial. El Programa estimó una ratio de 1 por cada 8/10 municipios, y estaba prevista la contratación de 20 dinamizadores rurales, de las que se materializaron 19. Entre las funciones de estos técnicos destacan la coordinación de la CTZ, la aplicación de metodologías, supervisión y seguimiento de las buenas prácticas identificadas, movilización de la ciudadanía, impulsor ejecutivo y responsable de transformar medios y presupuestos institucionales concretos. También desarrollar funciones de motivación, sensibilización y difusión a nivel político y social, con el fin de lograr un pacto por el empleo que comprometa a un apoyo real a las entidades y organizamos participantes.

En términos de la implementación de esta medida se observa un alto nivel de logro. El agente además, es visto como una figura potente que permitió hacer de enlace entre la comarca y la Diputación. Esto hace posible una mejor gestión de las intervenciones que se hacen desde el nivel central en el territorio y agilizar los flujos de información. Por tanto, se valora como muy relevante el estar físicamente en el propio territorio, condición que no se cumplía en algunos casos. 
Pero además de ejercer esta labor de puente, los agentes también son vistos como una figura cercana en el territorio; a diferencia del caso de los servicios centrales, que son considerados entes muy lejanos a las dinámicas locales de los territorios.

El programa establece que se deberán constituir, dentro de la estructura de cada Pacto, una comisión de género -como sexta medida- que actúe de manera transversal en todas las medidas del proyecto, asegurando al mismo tiempo una verdadera estrategia de igualdad de oportunidades. Esta comisión estará encargada de proponer un estudio diagnóstico de la situación de las mujeres en relación al empleo en los territorios donde se desarrollan los pactos. Además, deberá realizar un seguimiento que asegure la presencia de la perspectiva de género dentro de todos los ámbitos de actuación del Proyecto "Granada-Empleo". En algunos casos la comisión de género se constituyó exclusivamente sobre la base del tejido asociativo o un proyecto que existía en el territorio en esta materia. La relación de comisiones de género constituidas en los diferentes territorios es de diez del total de doce pactos. Algunas de las razones esgrimidas que justifican la no constitución de la comisión de género son la falta de personal técnico dispuesto a trabajar esta materia en el territorio y la carencia de tejido asociativo relacionado con la mujer. Por otra parte, se destaca como un resultado en sí mismo el esfuerzo por organizar y coordinar la presencia de diversos actores asociados a la temática de género dentro de la comarca.

El Proyecto "Granada-Empleo" define el fomento de la responsabilidad social territorial séptima medida- como un eje fundamental del trabajo en el ámbito de los pactos. Esta persigue que todos los agentes que actúan como actores contribuyan de modo planificado, activo y voluntario a la mejora del desarrollo socioeconómico y sostenible para el futuro.

Esta medida tiene dos componentes. Por una parte, la responsabilidad social corporativa y, por otro, el tema de la sostenibilidad. Respecto del primero, se plantea que desde cada pacto se pondrá en marcha un programa de responsabilidad social, dirigido a instituciones y empresas ubicadas en los territorios. Esta sub-medida se concretizará en la elaboración de un protocolo metodológico para la implicación de las empresas en los itinerarios integrados de inserción sociolaboral, y en la elaboración de unas guías de promoción de inserción. Los resultados de la implementación de esta medida se materializan en la participación de cuarenta y cuatro empresas y asociaciones empresariales en los pactos territoriales por el empleo. Solo en el caso de un pacto no se registra participación de este actor. En cuanto a la actuación relacionada con la sostenibilidad, los indicadores de implementación indican que se han firmado diez acuerdos respecto al medio ambiente en los doce pactos. Estos acuerdos han sido firmados por un promedio de diecisiete entidades. En definitiva, las actividades de sostenibilidad implementadas para la media de los doce pactos es de tres iniciativas. Este promedio no considera dos pactos que presentan un número muy por encima de las cifras del resto. En un caso las actividades diseñadas fueron veintidós y en el otro trece.

\subsection{La implementación de itinerarios integrados de inserción sociolaboral}

El proceso de selección de personas beneficiarias de los itinerarios de formación fue el primer paso en la implementación de este objetivo. Esta medida tuvo en cuenta los criterios del grado de adecuación al perfil preferente definido para cada especialidad formativa, la pertenencia a colectivos prioritarios (jóvenes, mayores de 45 años, parados de larga duración, cuidadores de personas dependientes, inmigrantes, personas con discapacidad, minorías étnicas, jóvenes que no han superado la enseñanza obligatoria, víctimas de la violencia de género, ex toxicómano/as, ex reclusos/as), y la adecuación al perfil personal y profesional de objetivos de inserción laboral por cuenta propia y cuenta ajena. 
Durante la implementación de esta medida, junto al equipo técnico contratado para gestionar la puesta en marcha de los itinerarios, también participaron los dinamizadores rurales que estaban vinculados a los pactos territoriales. Estos también cumplieron la labor de detección de necesidades formativas en el territorio, lo que permitió vincular las realidades locales con la puesta en marcha de itinerarios específicos que dieran cuenta de las necesidades locales de formación.

Tabla 1. Distribución por sexo y edad de los/as que terminaron el curso y grupo de comparación (en porcentaje vertical)

\begin{tabular}{|l|c|c|c|c|}
\hline \multirow{2}{*}{ Variables (sexo y edad) } & Terminaron el curso (N=245) & \multicolumn{2}{|c|}{ Grupo de comparación (N=341) } \\
\cline { 2 - 5 } & Hombre (\%) & Mujer (\%) & Hombre (\%) & Mujer (\%) \\
\hline 18 a 30 años & 7,3 & 25,3 & 8,3 & 26 \\
\hline 31 a 44 años & 5,7 & 34,7 & 5,6 & 33 \\
\hline 45 y más años & 1,2 & 25,7 & 1,8 & 25,4 \\
\hline
\end{tabular}

Fuente: Informe final de evaluación externa del Proyecto "Granada-Empleo", p. 89

El Programa "Granada-Empleo" establece como medida concreta la impartición de módulos de formación específica, como medio de favorecer la adquisición y mejora las cualificaciones y competencias profesionales, al mismo tiempo que hace posible su participación en prácticas profesionales tutorizadas. Respecto de la cantidad de acciones formativas realizadas, la implementación de este objetivo del programa tiene un alto nivel de logro $(96 \%)$, y la temporalización fue muy cercana a la prevista. En relación con el tipo de formación, los orientadores señalan la necesidad de tender hacia una pedagogía que integre de forma más sistemática la práctica con la teoría. Así mismo identifican la falta de homogeneidad en las características de los beneficios de un mismo curso, y su potencial impacto negativo en las acciones más técnicas. La valoración en general de los módulos es positiva -tanto de las acciones formativas (90,2\% de alternativas positivas) como de los conocimientos adquiridos (91,8\% categorías positivas)-, y se señala como un beneficio directo y visible para el territorio local, ya sea por su impacto en la formación, como por la potencial posibilidad de inserciones laborales.

La impartición de módulos de formación transversal y complementaria es una medida con carácter obligatorio para todas las personas beneficiarias directas del Proyecto. Los módulos transversales previstos son igualdad de oportunidades entre hombres y mujeres, nuevas tecnologías de la información y comunicación, sensibilización medioambiental, lucha contra la exclusión social y la no discriminación, y la prevención de riesgos laborales. Los cursos realizados se implementaron tal y como se establecía en el programa, y la evaluación de resultados muestra una alta valoración de los orientadores, que los consideran un apoyo fundamental a la búsqueda de empleo, con un impacto positivo indirecto en la cualificación del mercado de trabajo.

La segunda etapa de la formación consistía en la realización de prácticas profesionales tutorizadas. Estas tenían por objetivo potenciar la adquisición de competencias profesionales y situar a los estudiantes en un contexto laboral más cercano a lo que se podrían encontrar en la realidad. En cuanto a la implementación, ha habido menos beneficiarios y meses de prácticas que los programados originalmente por el proyecto, y la temporalización, en algunos casos, no se planificó considerando las características de los sectores económicos. No obstante, la evaluación de resultados muestra una alta valoración de las prácticas $(91 \%$ de valoraciones positivas). 
Otras de las medidas diseñadas y llevadas a cabo fueron la mejora de la calidad de los procesos formativos a través de la realización de una guía didáctica por cada una de las especialidades, y la gestión de becas y ayudas.

Las acciones de orientación e inserción sociolaboral, como última medida, tienen una doble tarea, la orientación sociolaboral, donde el orientador será la persona de referencia en el proceso de inserción al evaluar las potencialidades de los futuros beneficiarios, y la tutoría individual y su seguimiento, que consisten en entrevistas en profundidad con los beneficiarios donde se definan las características de sus procesos de inserción.

En cuanto a la implementación de esta medida, se detecta un satisfactorio nivel de cumplimiento de lo previsto en el Programa en referencia las acciones de orientación e inserción, y una crítica de los orientadores a la falta metodología y materiales de apoyo propiciados por el Programa. Uno de los fracasos más relevantes relacionados con los itinerarios de inserción, ha sido la incapacidad del Programa para generar puestos de trabajo por cuenta propia y empresas autónomas.

Por el contrario, uno de los logros del Programa es haber conseguido que al finalizar los itinerarios de formación 261 personas consiguieran empleo, frente a los 256 previstas. Así, el índice general de ocupación (división entre el $\mathrm{n} .{ }^{\circ}$ de alumnos formados ocupados y el total de alumnos formados) es de un $43 \%$-tres puntos sobre lo previsto-

Respecto de las personas que consiguen empleo en la misma acción formativa que han participado, el porcentaje del índice de coherencia ocupacional (división entre el n. ${ }^{\circ}$ de alumnos formados ocupadas "coherentemente" con el total de alumnos formados) es del $31,8 \%$ respecto del total de personas que han finalizado los itinerarios (606).

Tabla 2. Comparación entre quienes terminaron el curso y el grupo de comparación respecto de la situación laboral actual (en porcentaje vertical)

\begin{tabular}{|l|c|c|}
\hline & $\begin{array}{l}\text { Terminaron el curso } \\
(\%)(\mathrm{N}=245)\end{array}$ & $\begin{array}{l}\text { Grupo de comparación } \\
(\%)(\mathrm{N}=341)\end{array}$ \\
\hline Con empleo fijo & 11 & 9,4 \\
\hline Con empleo ocasional & 26,9 & 16,7 \\
\hline Trabajando por cuenta propia & 1,2 & 3,8 \\
\hline Trabajando sin contrato & 5,7 & 5 \\
\hline Desempleado & 49,4 & 57,8 \\
\hline Estudiando & 4,9 & 7,3 \\
\hline Otro & 0,8 & 0 \\
\hline
\end{tabular}

Fuente: Informe final de evaluación externa del Proyecto "Granada-Empleo", p. 136

Respecto de la inserción se observa en la tabla anterior que un 39,1\% de los beneficiarios encuestados declaró estar actualmente trabajando con contrato en el momento de hacer el estudio, ya sea con "empleo ocasional", "empleo fijo" o "por cuenta propia". Este porcentaje se acerca bastante al total de inserción conseguido por el Programa (40,7\%). Entre estas condiciones el porcentaje más elevado se lo lleva "empleo ocasional" $(26,9 \%)$ que por sí sola representa un $68,8 \%$ del total de inserción declarada. Si comparamos estos datos con la muestra de personas del grupo de comparación se observa un poco menos del $10 \%$ entre quienes declararon estar trabajando con contrato (29,9\%). Al igual que entre quienes terminaron la formación, en el caso del grupo de comparación son los que tienen un "empleo ocasional" los que reúnen un porcentaje mayor (16,7\%), aunque las diferencias son menores con el resto de condiciones laborales. En este caso esta condición representa el 55,9\% del total de personas que declararon tener empleo con contrato en la actualidad. 
Figura 2. Opinión sobre la utilidad de la formación (en porcentaje) $(\mathrm{N}=245)$

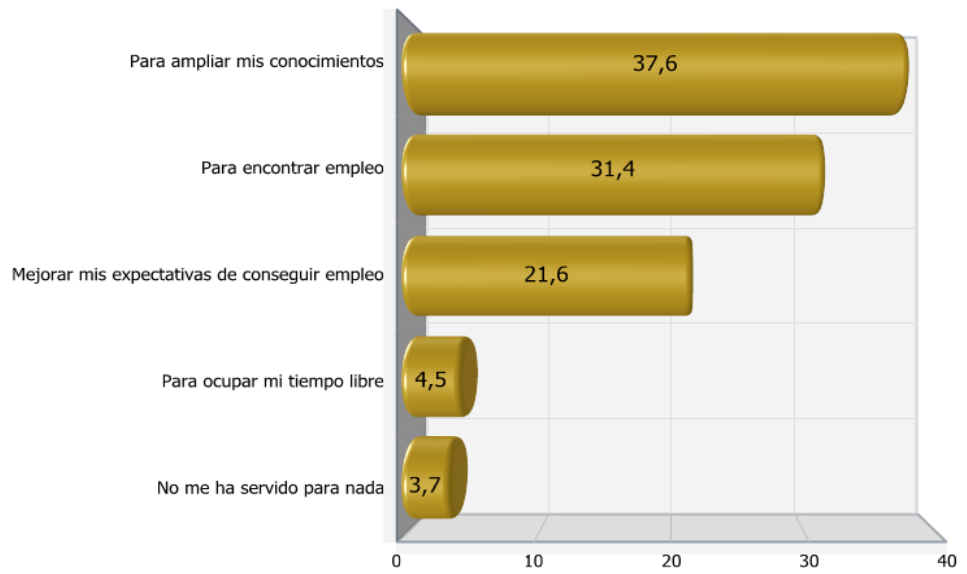

Fuente: Informe final de evaluación externa del Proyecto "Granada-Empleo", p. 144

En cuanto a la utilidad de los cursos de formación, un 53\% declara que el itinerario le han servido para modificar su situación laboral. De este grupo, un $61,5 \%$ se encontraba trabajando en el momento de la aplicación de la encuesta.

Figura 3. Aplicación de los conocimientos aprendidos en su trabajo (en porcentaje) ( $\mathrm{N}=96)$

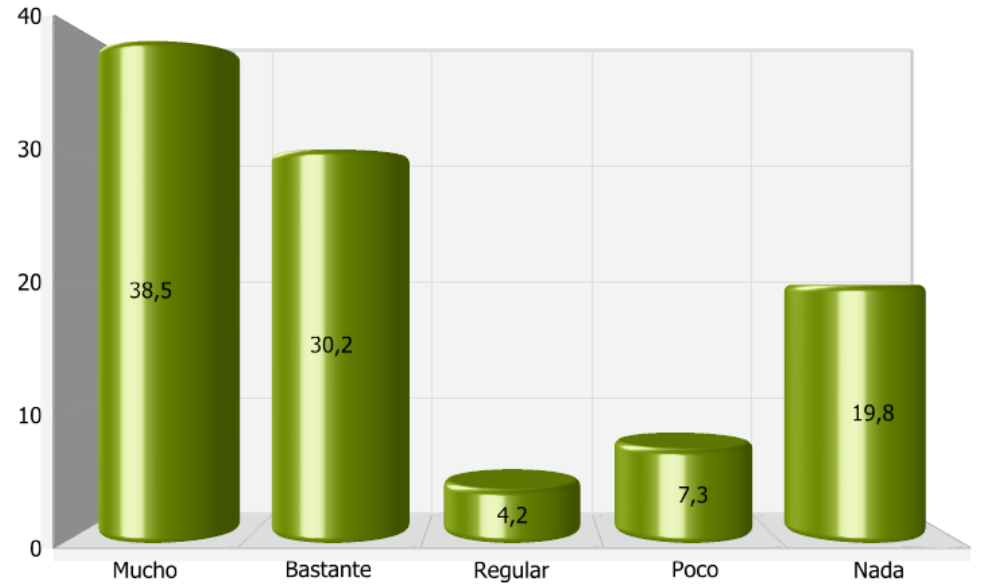

Fuente: Informe final de evaluación externa del Proyecto "Granada-Empleo", p. 145

Por otra parte, un tercio del total $(31,4 \%)$ cree que el curso le ha servido para "encontrar empleo", mientras que el $21,6 \%$ cree que ha "mejorado sus expectativas de conseguir empleo". De los que declararon estar trabajando, un 68,7\% declaró que los utilizaba los conocimientos aprendidos en el curso "mucho" o "bastante". A pesar de que el 27,1\% (suma de las categorías "poco" y "nada") declara que los conocimientos del curso no le han servido para nada en su trabajo actual. Así, el índice de utilización de los conocimientos aprendidos 
(división entre el $\mathrm{n}^{\mathrm{o}}$ de alumnos formados que utilizan habitualmente los conocimientos aprendidos en el curso y el total de alumnos formados) alcanza el 10,9\%.

Además de los análisis descriptivos se llevaron a cabo análisis estadísticos bivariados y multivariados de los grupos "terminaron la formación" y el "grupo de comparación", que consideraban como variable dependiente la situación laboral actual (empleado o desempleado), e independientes la motivación para realizar el curso, formación, sexo, edad, nivel de estudios y meses de desempleo. Las correlaciones bi-variables (Taub_b de Kendall y Rho de Spearman) y pruebas realizadas ( $Z$ y regresión logística) entre $\bar{l}$ los dos grupos comparados mostraron que un $96,8 \%$ de la condición de tener o no trabajo no es explicado por ninguna de las variables incluidas en el estudio.

\section{CONCLUSIONES}

La evaluación a la que se ha sometido al Proyecto "Granada-Empleo" ofrece un modelo participativo de los agentes políticos y técnicos que intervienen en la implementación del mismo, a partir de un modelo diseñado ad hoc, que ha conllevado la aplicación original de la propuesta de indicadores e índices de eficacia y eficiencia que se han expuesto en este trabajo.

En el marco del desarrollo del impulso y consolidación de los pactos territoriales por el empleo, podemos concluir que ha existido un esfuerzo sistemático por conocer las necesidades del territorio local. Dicho proceso se ha llevado a cabo mediante numerosas visitas en terreno a los agentes locales y reuniones preparatorias. Este conocimiento se ha plasmado en herramientas concretas, como los mapas relacionales (creados en once de los doce territorios), de gran utilidad tanto para el trabajo de los dinamizadores como para la definición de los itinerarios de formación.

No obstante, una de las cuestiones señaladas como negativas en este primer objetivo es el impacto negativo que supuso la carencia en términos políticos de un acuerdo marco a nivel provincial, que permitiera la participación de actores relevantes en el tema de empleo como son los Agentes Locales de Promoción y Empleo (ALPEs). Otro punto débil, fue el retraso en la formalización de la firma de los pactos, lo que produjo inconvenientes en el trabajo de los agentes locales. A diferencia de la presencia activa de los agentes en las Comisiones Técnicas de Zona (CTZ), el lado opuesto lo representó la escasa participación en la Comisión Institucional de Zona (CIZ).

Respecto a la promoción de concertación territorial, si bien uno de los logros del Programa es el apoyo político local conseguido en la firma de los doce pactos territoriales, el punto débil lo representa la escasa vinculación de la ciudadanía con el proyecto.

En lo que respecta a los dinamizadores rurales o agentes territoriales, existe entre las entidades públicas -en las que son vistos como los auténticos promotores del pacto- y privadas que participaron en Granada Empleo, un alto nivel de valoración de su trabajo (una puntuación general de 8,5 sobre 10 ).

Por último, el tema de la responsabilidad social territorial se tradujo en la firma de diez acuerdos de respeto al medio ambiente y la puesta en marcha de actividades de responsabilidad social, y fue abordado en los propios POAs, a través de medidas concretas sobre sostenibilidad.

Junto con el establecimiento de pactos territoriales, el otro gran objetivo del "GranadaEmpleo" es la implementación de itinerarios integrados de inserción sociolaboral. En este 
apartado, el proceso de selección de beneficiarios -que ha sobrepasado el número establecido de personas en un 10\%-, la implementación de los cursos de formación $-96 \%$ de porcentaje de realización-, y las prácticas profesionales han sido valoradas de modo muy positivo.

En cuanto a la inserción sociolaboral que logra el Programa, un $43 \%$ de las personas que terminaron la formación (606) declaran haber conseguido empleo después de terminado el itinerario (índice general de ocupación). Esto supone un porcentaje superior al previsto $(40 \%)$, con lo que podemos concluir que es un resultado muy satisfactorio. De este grupo un $66,7 \%$ vincula el haber encontrado empleo con la formación recibida. Un 31,8\% del total de formados/as han encontrado empleo en la misma acción formativa que han participado (índice de coherencia ocupacional). Un dato negativo de la inserción, sin embargo, es la inexistencia total de personas que consiguieron empleo por cuenta propia, esto a pesar de que fueron identificadas iniciativas de autoempleo en el marco del Programa.

La mayoría de los que encontraron empleo $(47,9 \%)$ tienen un contrato a tiempo parcial, mientras que un 33,3\% lo tiene a tiempo definido, y solo el 7,6\% tiene un contrato de más de 1 años de duración. Esto muestra que la inserción se relativiza si consideramos el factor de la calidad de empleo de los beneficiarios del Programa.

En cuanto a la utilidad del curso para los/as alumnos, el $22,9 \%$ considera que ha tenido una utilidad muy alta, ya que han conseguido empleo, utilizan los conocimientos aprendidos y declaran no haber podido conseguir ese empleo sin el curso. Para el 53\% el curso ha tenido una utilidad alta, ya que han conseguido un empleo, y o bien utilizan los conocimientos en el trabajo $(26,9 \%)$ o creen que la consecución de su puesto de trabajo se lo deben a la realización del curso $(26,1 \%)$. Sin embargo, para el $15,5 \%$ la formación ha tenido una utilidad media, ya que a pesar de no conseguir empleo, opinan que el curso les ha servido para mejorar sus expectativas de conseguirlo. Por último, para el 2,9\%, el curso ha tenido una utilidad nula, para estos no solo no han conseguido trabajo, sino que opinan que tampoco les ha aportado ninguna ventaja a la hora de conseguirlo.

Entre las recomendaciones finales hechas a las autoridades y técnicos implicados tras la evaluación del Programa, se recomienda, entre otras cuestiones, (1) el progreso hacia la concreción de un acuerdo político marco a nivel provincial sobre empleo, que permita la participación de todos los actores involucrados en los territorios locales; (2) la complementación de la formación teórica con la práctica en un mismo espacio formativo, (3) la potenciación de las estrategias de intervención que hagan posible una inserción laboral de mayor calidad, y (4) el apoyo a las iniciativas de autoempleo identificadas en el marco del Programa.

\section{BIBLIOGRAFÍA}

CACHÓN, L. (2004) (dir.), Colectivos desfavorecidos en el mercado de trabajo y políticas activas de empleo. Madrid, Ministerio de Trabajo y Asuntos Sociales.

CANALES, J.M. (2002), "La evaluación de las políticas públicas y de las organizaciones públicas", en J.M. CANALES, Lecciones de administración y de gestión pública. Alicante, Universidad de Alicante, pp. 191-212

DELGADO, S. y ORTEGA-PÉREZ, N. (coords.), Políticas activas de empleo en Andalucía. Granada, Comares (en prensa).

FEMP (2005), Aspectos de la intervención de las administraciones públicas locales en las políticas activas de empleo en España. Madrid, Ministerio de Trabajo y Asuntos Sociales. 
PÉREZ, M. (2011), "La evaluación de las políticas de empleo: necesidad, estrategias y metodologías de la formación profesional", III Congreso Nacional de la Red Española de Política Social, Pamplona (sin publicar).

RIVERO, A. (2003), Evaluación de políticas activas de empleo: el caso de las formas de intervención cofinanciadas por el Fondo Social Europeo en España. Madrid. Ministerio de Trabajo y Asuntos Sociales.

SUBIRATS, J. (2005), "Catorce puntos esenciales sobre evaluación de políticas públicas con especial referencia al uso de las políticas sociales", EKonomiaz, n ${ }^{\circ}$ 60, vol. I, pp.18-37.

TRINIDAD, A. y PÉREZ, M. (2010), Análisis y evaluación de políticas sociales. Madrid, Tecnos. -- (1998), "Evaluación de programas de intervención social". Revista del Ministerio de Trabajo y Asuntos Sociales, 10, pp.113-140.

\section{Breve currículo}

\section{Margarita Pérez Sánchez}

Licenciada en Ciencias Políticas (sección Ciencia Política) y licenciada en Sociología (sección Sociología Política), Universidad Complutense de Madrid. Diplomada en Derecho Constitucional, Centro de Estudios Constitucionales de Madrid. Diplomada en Inglés por el Instituto de Idiomas de la Universidad Complutense, Curso de especialización en Administración Pública por la London School of Economics and Political Science y doctora en Ciencias Políticas y Administración, Universidad de Granada. Ha cursado estudios de Técnicas de Investigación y Estadística en la Universidad de Essex (Reino Unido) y ha sido profesora visitante en las Universidades de Edimburgo (Reino Unido), Trinity College (Irlanda) y Notre Dame (EE.UU.), investigadora en: Roma (ISFOL), Berlín (CEDEFOP) y Dublín (FAS). Líneas de investigación: Análisis y evaluación de políticas públicas.

\section{Antonio Trinidad Requena}

Doctor en Sociología por la Universidad de Granada. Catedrático de Sociología de la Universidad de Granada y Director del Grupo de Investigación "Problemas Sociales en Andalucía". Ha sido Visiting Scholar en la London School of Economics and Political Science. Publicaciones se encuentran los libros Teoría fundamentada "Grounded Theory". La construcción de la teoría a través del análisis interpretacional; Servicios sociales: planificación y evaluación; Leer la sociedad; La realidad económica y social de las personas mayores y Administración pública y estado de bienestar. Entre sus últimos proyectos de I+D destaca "Relocalización industrial e inmigración: el papel de la industria exportadora en los países de origen (Marruecos y México)".

\section{Nieves Ortega Pérez}

Doctora en Ciencia Política y de la Administración: Universidad de Granada. Profesora en las Universidades de Granada y Jaén, en los Departamentos de Ciencia Política y de la Administración, y del Departamento de Derecho Público y Privado Especial respectivamente. Ha formado parte del Center for Comparative Immigration Studies de la Universidad de California, San Diego, como guest scholar, y ha sido visiting research fellow en el Sussex Center for Migration Research de la Universidad de Sussex en el Reino Unido. También ha realizado estancias de investigación en la Unité de Recherche Migrations et Société (Université de Paris 7) y en la Université de Québec à Montréal. Ha publicado múltiples artículos en revistas nacionales e internacionales y capítulos de libro. Líneas de investigación: Análisis de políticas públicas, en concreto: políticas de inmigración, y procesos y sistemas políticos en América Latina y el Magreb. 